\title{
MATCH PROBABILITY STATISTICS AND GAMMA RAY BURST RECURRENCES IN THE BATSE CATALOG
}

\author{
SUN HONG RHIE \\ Institute of Geophysics and Planetary Physics, Lawrence Livermore National Laboratory, \\ Livermore, CA 94550, U.S.A \\ and \\ DAVID BENNETT \\ Center for Particle Astrophysics, University of California, Berkeley, CA 94720, U.S.A.
}

\begin{abstract}
We develop match probability statistics to test the recurrences of gamma ray bursts in the BATSE catalog $1 \mathrm{~B}$ and $2 \mathrm{~B}$. We do not find a signal of repetitions at the match level of $10^{-3}$.
\end{abstract}

Key words: Gamma ray bursts - Recurrences

It is an interesting question whether classical gamma ray bursts repeat, especially because of the potential implication on the distance of the sources. Gamma ray bursts were first discovered [1] by Vela satellites in 1967 the same year the first radio pulsar was discovered. Pulsars are understood to be rotating neutron stars in essence, but the origin of gamma-ray bursts is a complete mystery. If they are from the (extended) Galactic halo, the isotropic emission energy will be $\sim 10^{43} \mathrm{ergs}$. If they are from cosmological distances, the isotropic emission energy will be $\sim 10^{51} \mathrm{ergs}$. For example, the bursts from cosmological distances can not be repetitive because the enormous energy output would most likely be accompanied by the consumption of the sources.

Currently, we do not have dynamic clues toward a more meaningful identification or rejection of repetitions, and the controversy lies in whether there is statistical evidence of repeating classical gamma ray bursts in the BATSE catalog. In order to address the problem, we ask ourselves the following questions.

1. Can we define a quantity which measures the probability for a given pair of GRB events to have come from the same position of the sky?

2. Can we find a statistic which can gauge the significance of matched pairs?

3. Is there a statistically significant excess of matched pairs in the BATSE catalog?

As an answer, we construct the 'match probability' of a given pair of events based on measurement error distribution functions, build statistics using 'the match probability', and analyse the publically available BATSE 1B and $2 \mathrm{~B}$ data sets.

Our statistics is built based on the simple observation that the repetitive bursts from the same source form an error distribution in the position space. 
An obvious but important aspect of it is that it is highly localised (around the true source position). Gamma ray bursts in the BATSE catalog are known to be largely isotropic, and the BATSE catalog can be considered as a mixture of error distributions and a background isotropic distribution. An isotropic distribution is characterized by the complete lack of localization. The drastic difference in localization behavior of the two types of distributions is the underlying basis of our burst pair match probability statistics.

Given a pair of bursts, the probability that they have come from the same source depends on the joint probability density of the error functions of the events $f\left(\mathbf{x}_{1}\right)$ and $f\left(\mathbf{x}_{2}\right)$.

$$
\rho_{12}=\int_{S^{2}} f_{1}\left(\mathbf{x}_{1}\right) f_{2}\left(\mathbf{x}_{2}\right)=\int_{S^{2}} f_{1}\left(\mathbf{x}-\mathbf{x}_{1 c}\right) f_{2}\left(\mathbf{x}-\mathbf{x}_{2 c}\right) d^{2} \mathbf{x}
$$

where $\mathbf{x}_{1 c}$ and $\mathbf{x}_{2 c}$ are the center positions. If we assume gaussian error distributions (in $\Re^{2}$, which is good enough here),

$$
\rho_{12}=\frac{1}{2 \pi \sigma_{12}^{2}} e^{-r_{12}^{2} / 2 \sigma_{12}^{2}}
$$

where $\sigma_{12}^{2}=\sigma_{1}^{2}+\sigma_{2}^{2}$. We define the 'match probability' of the pair by integrating $\rho_{12}$ from the measured distance $r_{12} \equiv\left|\mathbf{x}_{1}-\mathbf{x}_{2}\right|$ to infinity.

$$
P_{12} \equiv P\left(r_{12}, \sigma_{12}\right)=\frac{1}{\sigma_{12}^{2}} \int_{r_{12}}^{\infty} e^{-r_{12}^{2} / 2 \sigma_{12}^{2}} r d r=e^{-r_{12}^{2} / 2 \sigma_{12}^{2}}
$$

Now, we look at the distribution of pairs in $P$-space. For an error distribution in position space, the pair distribution in $P$-space is homogeneous. In other words, the number of pairs is a constant function of $P$. Since $P \in[0,1]$, the constant is the total number of pairs. By normalizing by the total number of pairs, we define the pair density distribution $g(P)$.

$$
g_{\mathrm{err}}(P)=1 . \quad ; \quad P \in[0,1]
$$

For the background isotropic distribution,

$$
g_{\text {iso }}(P) \propto \frac{\sigma^{2}}{P} .
$$

(The number of pairs at a distance $r \propto r d r$ and $r$ and $P$ are related by Equation (3.) It is not surprising that $g_{\text {iso }}(P)$ is highly concentrated toward small $P$ because isotropic distribution has many more pairs at large separations in comparison to an error distribution of the same number of bursts.

$\sigma<<1$ for most of the bursts in the BATSE catalog, and $g_{\mathrm{err}}(P)>$ $g_{\text {iso }}(P)$ unless $P$ is very small. Thus, we define the following statistical quantities.

$$
\Delta Q_{\alpha} \equiv Q_{\alpha}(p)-<Q_{\alpha}(p)>_{\text {iso }} \quad ; \alpha=0,1, \ldots
$$


$Q_{\alpha}(p)=\sum_{i<j} P_{i j}^{\alpha} \Theta\left(P_{i j}-p\right)$

where $\Theta(x)=1$ if $x>0$ and $\Theta(x)=0$ otherwise. The signal should be found in $\Delta Q_{\alpha}$, and the background isotropic distributions for $\left\langle Q_{\alpha}(p)\right\rangle_{\text {iso }}$ are generated by randomizing the burst positions while keeping the error functions of the data. In the limit of a large sample, we can calculate $\Delta Q_{\alpha}(p)$.

$$
\Delta Q_{\alpha \mathrm{th}}(p)=\frac{N_{\text {pairs }}}{\alpha+1}\left(1-p^{\alpha+1}\right)-\sum_{\text {matched pairs }} \frac{\sigma_{i j}^{2}}{2} .
$$

Therefore, we can calculate the expected signal to noise ratio $(S / N)$ by comparing $\Delta Q_{\alpha \text { th }}(p)$ to the RMS fluctuations about $<Q_{\alpha}(p)>$ iso. We find that $Q_{1}(0)$ has the highest $S / N$. For the fraction of the matched pairs (of bursts $\in$ error distribution) of $10^{-3}$, the $S / N$ of $Q_{1}(0) \approx 3.0$ for 485 bursts (exclusive of the events without error estimations) of BATSE 2B. We emphasize that our 'match probability' test of the BATSE catalogs for repetition is at the match level of $\approx 10^{-3}$. For example, if five bursts in the BATSE $1 \mathrm{~B}$ are from a repeater as suggested by Wang and Lingenfelder [6], the signal can not be seen statistically because the required sensitivity is $\approx 3 \times 10^{-4}$. The analyses of $Q_{1}(0)$ statistics of the BATSE data are shown in Table 1.

TABLE I

The number of matches and anti-matches measured with the $Q_{1}(0)$ statistic with 1- $\sigma$ error bars determined from the RMS deviation from the mean of 10,000 simulated BATSE catalogs. The significance refers to the fraction of the 10,000 simulated catalogs with larger values of $Q_{1}(0)$ than the real data.

\begin{tabular}{rrrrr}
\hline Sample: & All & $\sigma_{\text {stat }}<9^{\circ}$ & $\sigma_{\text {stat }}<4^{\circ}$ & Exposure Cut \\
\hline$N_{\text {match 1B }}$ & $13 \pm 25$ & $27 \pm 14$ & $1 \pm 7$ & $3 \pm 12$ \\
$N_{\text {antipode 1B }}$ & $54 \pm 25$ & $24 \pm 14$ & $17 \pm 7$ & $9 \pm 12$ \\
match signif. 1B & $29.3 \%$ & $2.65 \%$ & $43.36 \%$ & $36.96 \%$ \\
antipode signif. 1B & $2.42 \%$ & $4.54 \%$ & $1.32 \%$ & $21.92 \%$ \\
\hline$N_{\text {match 2B }}$ & $-23 \pm 45$ & $0 \pm 26$ & $-13 \pm 13$ & $-9 \pm 20$ \\
$N_{\text {antipode 2B }}$ & $31 \pm 45$ & $39 \pm 26$ & $29 \pm 13$ & $1 \pm 20$ \\
match signif. 2B & $68.31 \%$ & $48.33 \%$ & $82.39 \%$ & $65.61 \%$ \\
antipode signif. 2B & $23.34 \%$ & $7.84 \%$ & $1.90 \%$ & $46.72 \%$ \\
\hline
\end{tabular}

We conclude that there is no signal of recurrent bursts in the BATSE data at the match level of $10^{-3}$. For more details, see [2]. 
Now, where does our statistic lead to in regard to the current controversy on the repetition of classical gamma ray bursts? The outcome of devising a new statistic may not be of much value if it merely provides a third statistic and another opinion on the issue. It turns out that we can understand both two-point correlation [4] and nearest-neighbor statistics [3] from 'match probability' statistics because of the fuller position measurement information contained in it and moreover we can estimate the $S / N$ of the statistics. Two point correlation function is directly related to $Q_{0}(p)$ and the $S / N$ at the optimal $p_{\text {op }}$ is somewhat worse than the true optimal case of $Q_{1}(0)$. One should keep in mind that the optimal value $p_{\text {op }}$ can be found only by embedding the two point correlation function in the match probability statistics. In order to understand the nearest neighbour statistics (NNS), we studied the cases of 60 matched pairs. We find that NNS has slightly better sensitivity than $Q_{1}(0)$ when each source bursted only twice. For multiplicities bigger than two, the $S / N$ of NNS degrades dramatically and is far worse than that of the two point correlation statistics. Therefore, the conjecture by Quashnock and Lamb [3] based on nearest neighbour correlations that there are recurrent bursts with high multiplicities in the BATSE catalog is not supported by more sensitive tests. NNS also has a peculiar disadvantage that the sensitivity does not improve as the size of the sample grows.

Finally, we would like to comment on the 'signal' Quashnock and Lamb [3] and Narayan and Piran [4] have seen. Is the 'signal' identified in our analyses? Yes: In the sample $\sigma_{\text {stat }}<9^{\circ}$ ( without exposure corrections), the curve $Q_{1}(p)$ follows that of $Q_{1 \text { iso }}$ and then deviates from it at around $p=0.6$ to follow the (theoretical) curve of signal for $10^{-3}$ matches. Speaking in terms of $g(P)$, there is a fluctuation (depletion and excess) in $g(P)$ at around $P=$ 0.6 compared to that of $g_{\text {iso. }}$. Is the 'signal' consistent with the expectation from repeating bursts? Clearly, it is not. What is this 'signal' a signal of? We do not know. It could be due to selection effects $[4,5]$ or many other reasons as yetuntested. But it is also consistent with statistical fluctuations. When we make exposure corrections using the trigger efficiencies provided in the BATSE catalog, the 'signal' disappears. We note that the events excluded by this cut are mostly faint bursts.

\section{References}

1. Klebesadel, R., Strong, I.B., and Olson, R. A.; 1972, Astrophys. J. 185, L85.

2. Bennett, David P, and Rhie, Sun Hong ; 1994, Astrophys. J. submitted.

3. Quashnock, J.M. and Lamb, D.Q.; 1993, M.N.R.A.S. 265, L59.

4. Narayan, R., and Piran, T.; 1993, M.N.R.A.S. 265, L65, and references therein.

5. Maoz, E.; 1994, M.N.R.A.S. 269, L5.

6. Wang, V. C., Lingenfelter, R. E.; 1995 Astrophys. J. 441. 